\title{
Heart Attack in a Young Woman-All About Genetics!
}

\author{
Sara Lemos Rocha ${ }^{1}$, Diana Ferreira ${ }^{1}$, Bruno Serra $^{1}$, Vitor Costa $^{1}$ and Carla Veiga Rodrigues ${ }^{2}$ \\ 1. Intern of General Practice and Family Physician on Unit of Primary Health Care B, Chaves 5400, Portugal \\ 2. Intern of General Practice and Family Physician on Unit of Primary Health Care S. Neutel, Chaves 5400, Portugal
}

\begin{abstract}
INTRODUCTION: Cardiovascular diseases, such as acute myocardial infarction (AMI), are the leading causes of mortality and morbidity in the world. The pathophysiological mechanisms of AMI are multiple, but 50\% results from thrombus derived from an atherosclerotic plaque rupture. The role of factor $\mathrm{V}(\mathrm{fV})$ mutations in the arterial disease is controversial, but several studies suggest that this mutation can contribute to AMI in young people and in those with adjuvant cardiovascular risk factors. CASE DESCRIPTION: Caucasian woman, 36 years old. Family history of early death due to AMI from maternal and paternal origin. Father deceased at the age of 44 from a stroke. Admitted at the hospital presenting AMI symptoms, which was confirmed. During her admittance genetic study of prothrombotic mutations were requested, which revealed a heterozygous fV Leiden mutation. CONCLUSION: The association between $\mathrm{fV}$ mutation and arterial events is controversial, but one of the studies showed that the heterozygous $\mathrm{fV}$ mutation is associated with a significantly increased risk of AMI in 2-3 times, especially if cardiovascular risk factors coexist. The Family Physician plays a key role in the management of chronic diseases, especially in young patients, due to its impact on everyday life and the necessary changes in lifestyle.
\end{abstract}

Key words: Acute myocardial infarction (AMI), fV mutation, arterial disease, young woman.

\section{Introduction}

Cardiovascular diseases, such as acute myocardial infarction (AMI), are the leading causes of mortality and morbidity worldwide. Genetic and environmental factors contribute to AMI [1].

The pathophysiological mechanisms of AMI are multiple, but $50 \%$ results from thrombus derived from an atherosclerotic plaque rupture. The rupture of the atherosclerotic plaques and subsequent thrombosis are complications of advanced stage atherosclerotic lesions, whose rupture activates blood coagulation cascade, initiating thrombus formation and subsequent occlusion of the artery.

The role of the Factor V Leiden (fV) mutation in arterial disease is controversial, but several studies suggest $\mathrm{fV}$ mutations can contribute to AMI in young people and in those with cardiovascular risk factors (CVRF). Mansourati et al. [2] state in their work that factor V Leiden was found in $12 \%$ of young patients (mean age 44 years) with Myocardial infarctions with

Corresponding author: Sara Raquel Lemos Rocha, M.S. normal (or near-normal) coronary arteries, in $4.5 \%$ of patients with AMI and significant coronary artery disease, and in $5 \%$ of normal controls.

Factor V Leiden thrombophilia is characterized by a poor anticoagulant response to activated protein $\mathrm{C}$ (APC) [3]. The prevalence of mutation ranges from $2 \%$ to $15 \%$ on general population and it is the most frequent genetic cause of venous thrombosis on Caucasians [4].

The thrombophilia should be suspected on individuals with a previous history of venous thromboembolism (VTE), particularly in women with a history of VTE during pregnancy or associated with oral contraceptive pills, and in individuals with a personal or family history of recurrent thrombosis [5-7].

Heterozygosity for the factor V Leiden allele and the associated risk for venous thrombosis are inherited in an autosomal dominant manner. Homozygosity for the factor V Leiden allele and an even greater risk for venous thrombosis are inherited in an autosomal recessive manner. 
The diagnosis of factor $\mathrm{V}$ Leiden thrombophilia is made either using a coagulation screening test or by DNA analysis of $F 5[8,9]$.

The anticoagulant treatment is indicated during acute phase after the thrombotic event. The maintenance of anticoagulation is evaluated, taking into account recurrence and bleeding risk.

The present work reports the case of a Caucasian woman, who had an acute myocardial infarction at the age of 36 years old.

\section{Case Description}

Caucasian woman with 37 years old, dyslipidaemia controlled with diet and exercise, and taking oral contraceptive pills for 17 years.

Her family history includes 2 grandparents (maternal grandfather and paternal grandmother) deceased by AMI at the age of 60 and her father passed away at the age of 44 from a stroke. The family's genogram is presented on Fig. 1.

At 36 years old she was admitted to the hospital presenting AMI clinic, confirmed by EKG and blood tests. During her stay at the hospital, a genetic study of prothrombotic mutations was performed, revealing a heterozygous fV Leiden mutation. She was discharged medicated with double anti-aggregation and with indication to change the contraceptive method.

She was advised to do prophylaxis of thromboembolism with Low Molecular Weight Heparin (LMWH) in situations of increased thromboembolic risk, such as pregnancy, post-op situations, bed confinement pathologies and prior to long trips with inferior member's immobilization for more than six hours.

Her husband suffered an ischemic stroke at 38 years old and is still under study at the moment, presenting a normal prothrombotic study at the moment.

A genetic study was also carried out on her daughters, revealing an $\mathrm{fV}$ mutation on her younger daughter's test and on her sister, whom is still under study.

The 9 year-old daughter (older) has thrombophilia without $\mathrm{fV}$ Leiden mutation and the four year old has thrombophilia with heterozygous fV Leiden mutation.

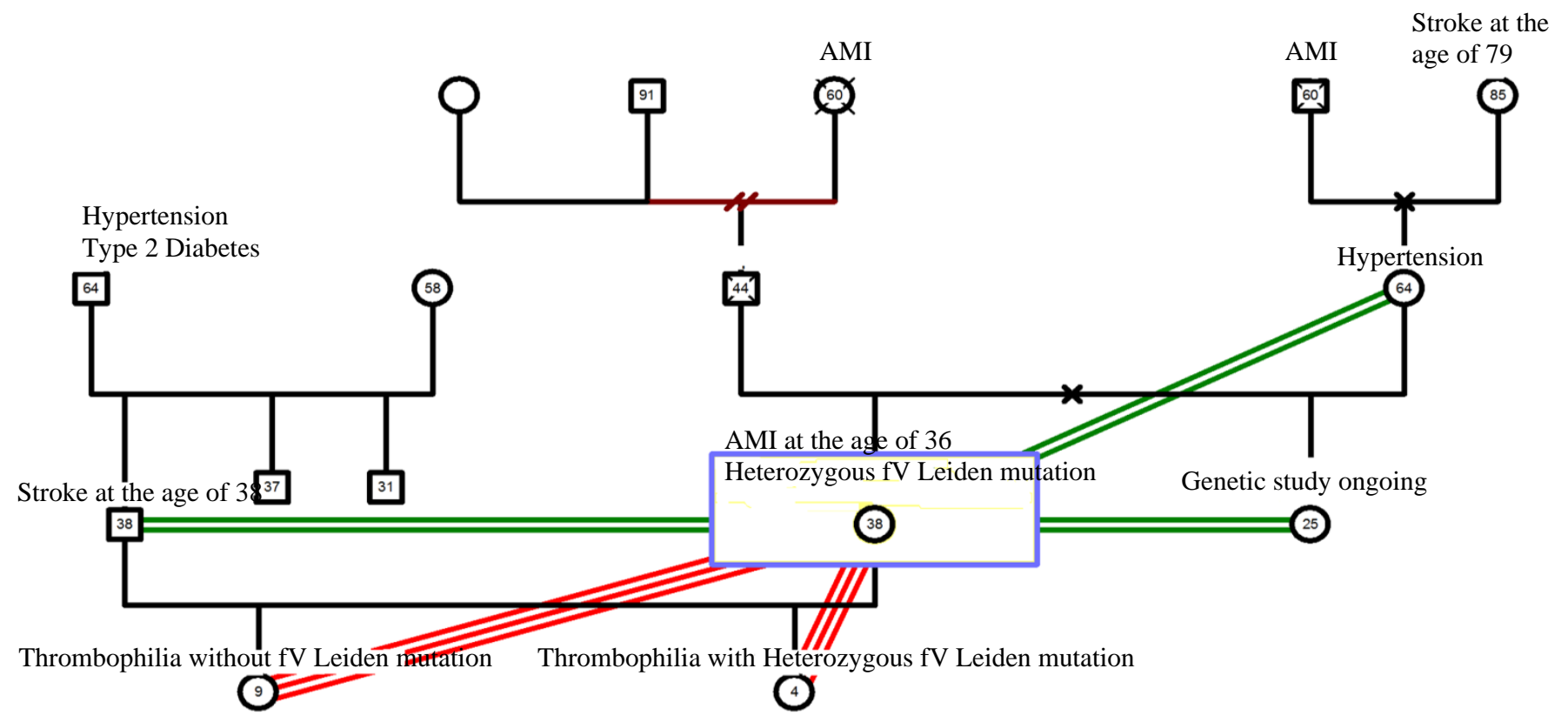

Fig. 1 Genogram. AMI-Acute Myocardial Infarction; fV-factor V. 


\section{Conclusion}

The association between $\mathrm{fV}$ mutation and arterial events is controversial, but some studies showed that the heterozygous $\mathrm{fV}$ mutation is associated with a significantly increased risk of AMI in 2-3 times, especially if there is coexistence of cardiovascular risk factors.

Some studies have reported positive associations between $\mathrm{fV}$ Leiden mutations and AMI, but others have not found any association.

Family Physicians play a key role in the management of chronic illness, particularly in young patients, due to its impact on everyday life and the necessary changes in lifestyle.

It is also crucial to help the family manage this hereditary event and cope with the possible fatalities it can enclose.

\section{References}

[1] Paludo, C. 2003. "Study of Genetic Risk Factors for Acute Myocardial Infarction at Young Age." Ph.D. thesis, Rio Grande do Sul University.

[2] Holm, J., Hillarp, A., Zoller, B., Erhardt, L., Berntorp, E., and Dahlback, B. 1999. "Factor V Q506 and Prognosis after Acute Coronary Syndrome." Thrombosis and Haemostasis 81 (6): 857-60.

[3] Rosendaal, F. R., Siscovick, D. S., Schartz, S. M., Beverly, R. K., Psaty, B. M., Longstreth, W. T., Raghunathan, T. E.,
Koepsell, T. D., and Reitsma, P. H. 1997. "Factor V Leiden (Resistance to Activated Protein C) Increases the Risk of Myocardial Infarction in Young Women.” Blood 89 (8): 2817-21.

[4] Rosendaal, F. R., Siscovick, D. S., Schwartz, S. M., Psaty, B. M., Raghunathan, T. E., and Vos, H. L. 1997. “A Common Prothrombin Variant (20210 G to A) Increases the Risk of Myocardial Infarction in Young Women." Blood 90 (5): 1747-50.

[5] Mansourati, J., da Costa, A., Munier, S., Mercier, B., Tardy, B., Ferec, C., Issaz, K., and Blanc, J-J. 2000. "Prevalence of Factor V Leiden in Patients with Myocardial Infarction and Normal Coronary Angiography." Thrombosis and Haemostasis 83 (6): 822-5.

[6] Bertina, R. M., Koleman, B. P. C., Koster, T., Rosendaal, F. R., Dirven, R. J., de Honde, H., van der Velden, P., and Reitsma, P. H. 1994. "Mutation in Blood Coagulation Factor V Associated with Resistance to Activated Protein C." Nature 369 (6475): 64-7.

[7] Jeffery, S., Leatham, E., Zhang, Y., Carter, J., Pratel, P., and Kaski, J. C. 1996. "Factor V Leiden Polymorphism in Patients with Ischaemic Heart Disease and in Different Populations Groups.” Journal of Human Hypertension 10 (6): 433-4.

[8] Gaspar, J., Benchimol, C., Gadelha, T., and Penna, G. 2011. "Arterial Thrombosis and Acute Myocardial Infarction with Angiographically Normal Coronary Arteries in a Woman Heterozygous for Both Factor V Leiden and Prothrombin Mutation." Portuguese Journal of Cardiology 30 (9): 727-9.

[9] Koeleman, B. P. C., Reitsma, P. H., and Bertina, R. M. 1997. "Familial Thrombophilia: A Complex Genetic Disorder." Semin Hematol. 34 (3): 256-64. 